\title{
Nasal Spreading as Defective Gestural Deactivation
}

\author{
Caitlin Smith \\ University of Southern California
}

\section{Introduction}

Nasal spreading (also called nasal vowel-consonant harmony) is a process by which a nasal segment in a word triggers nasalization of its surrounding segments. This paper proposes a reanalysis of this phenomenon using gestures as the units of phonological representation in order to more accurately capture its crosslinguistic typological patterns. Nasal spreading proceeds unboundedly, potentially resulting in spreading of nasality throughout an entire word. However, it is also common for the spread of nasality to be arrested by glides, liquids, or obstruents, a property known as blocking. This is exemplified in Warao (isolate; Venezuela), in which nasality spreads progressively from a triggering nasal consonant or vowel, affecting following vowels, glides, and glottal consonants (1a-d) but is arrested by liquids and obstruents, as in (1e-h) (Osborn 1966):
a. [mõ̃̃õ] 'cormorant'
e. [honĩ̃̃ãku] 'turtle'
b. [inã̃̃ã $\tilde{a}]$ 'summer'
f. [nãõote] 'he will come'
c. [mõãũ] 'give it to him'
d. [ㄸũãñ̃] 'pink eye'
g. [mõãũpu] 'give them to him!'
h. [õ̃ĩhõro] 'kind of tree'

It is also possible for certain consonants to fail to undergo nasal spreading while still allowing nasality to spread beyond it, a property known as transparency. An example of a system of nasal spreading that exhibits obstruent transparency is Tuyuca (Tucanoan; Colombia and Brazil), in which nasality is a property of an entire morpheme and is expressed on all segments except voiceless obstruents, which neither undergo nasal spreading nor block it from spreading further (Barnes \& Takagi de Silzer 1976; Barnes 1996).
a. [mĩpĩ] 'badger'
e. [j̃ãmĩ] 'night'

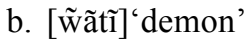
f. [พ̃̃̃̃õ] 'wind'
c. [jũũã] 'yucca soup'
g. [t̃̃yõ] 'Yapara rapids'
d. [jõõõ] 'bird'
h. [jõ̃̃̃̃] 'small hen'

Nasal spreading and the patterns in which segments undergo, block, or are transparent to it have been the focus of extensive and varied phonological study. Typically, spreading is represented as the addition of association lines between a spreading feature and the segments in a word, following the tenets of Autosegmental Phonology (Goldsmith 1976/1979, 1990). This paper, however, aims to reanalyze nasal spreading within the gesture-based framework of Articulatory Phonology (Browman \& Goldstein 1986, 1989, et seq.; henceforth AP) in order to address various issues that have arisen in the many treatments of this phenomenon in traditional feature-based theory. By proposing novel solutions to these issues, a typology of nasal spreading emerges that matches crosslinguistic facts more closely than previous accounts. This paper focuses on the analysis of blocking and transparency as two distinct mechanisms within nasal spreading and the predictions this makes about which segments may behave transparently or as blockers. In addition, it re-characterizes the source of the imperative to spread nasality as a property of the spreading element itself and examines the predictions this approach makes.

\footnotetext{
* Many thanks to Rachel Walker, Louis Goldstein, Khalil Iskarous, and Karen Jesney for all of their time and helpful advice. Thanks also to the attendees of Phonology 2014 for their comments and questions, and to the members of Phonlunch at USC and the weekly breakfast meeting for listening to repeated presentations of this work.
}

(C) 2015 Caitlin Smith

Proceedings of $A M P 2014$

Completed March 1, 2015 


\section{Defective Gestural Deactivation}

The aim of this paper is to reanalyze nasal spreading as the result of extended gestural duration. In AP, gestures are spatiotemporal units of phonological representation that specify certain goal vocal tract variables to be achieved in a certain amount of time during which the gesture is active. The gesture responsible for nasality is one with a goal tract variable of a lowered velum. During a velum opening gesture's activation interval, it exerts control over the velum in order to lower it to its goal position. After a specified period of time a typical gesture automatically deactivates and the velum is allowed to return to its neutral position. This paper proposes that nasal spreading is the result of a defective velum opening gesture's failure to automatically deactivate at a specified time, resulting in its extended duration and the nasalization of any other gestures with which it overlaps in time.

Unlike segments, features, or autosegments whose durations are specified only at the level of abstract, discrete timing slots, gestures unfold over a period of real time and may overlap one another. By manipulating that period of time over which a gesture unfolds, it becomes possible to model spreading phenomena within a gestural framework. Specifically, nasal spreading is modeled here as the extended duration of a velum opening gesture, resulting in its overlap with and nasalization of the gestures that follow. This proposal is demonstrated in the following figure, which shows a gestural score for the Warao form [mõjõ ] 'cormorant.'

\begin{tabular}{|c|c|c|c|}
\hline $\mathrm{m}_{1}$ & $\tilde{\mathrm{o}}_{2}$ & $\tilde{J}_{3}$ & $\tilde{\mathrm{o}}_{4}$ \\
\hline \multicolumn{4}{|l|}{ Velum open $_{1}$} \\
\hline Labial closure $_{1}$ & & $\begin{array}{c}\text { Tongue Body } \\
\text { palatal narrow } \\
\end{array}$ & \\
\hline \multicolumn{2}{|c|}{ Tongue Body uvular-pharyngeal narrow ${ }_{2}$} & Tongue $\mathrm{B}$ & geal narrow ${ }_{4}$ \\
\hline
\end{tabular}

Figure (1): A gestural score for the form [mõjõ] 'cormorant.' Subscript numbers indicate gestures' linear ordering indexations.

In a gestural score, the horizontal axis represents time, and each box's horizontal length represents the period of time over which a gesture is active, i.e., when it has control over the articulators in order to achieve its specified articulatory task. In [mõjõ], the [m] is represented by two gestures, one for closure of the lips and one for opening of the velum. The two vowels are represented by tongue body gestures specified for narrowing in the uvular-pharyngeal region, and the glide [j] by a tongue body gesture specified for narrowing in the palatal region. Of note here is the fact that the velum opening gesture of the $[\mathrm{m}]$ has an extended duration such that it overlaps all following gestures, resulting in their nasalization. The dashed line within the velum opening gesture indicates the time at which a typical velum opening gesture would deactivate. This is the source of nasal spreading in Warao: nasal consonants and vowels are accompanied by velum opening gestures that extend in duration beyond what is typical for a velum opening gesture in a language without nasal spreading.

The question now turns to what is driving the extended activation duration of the velum opening gesture as seen in the gestural score. Following Smith (forthcoming), this work assumes that the phonological component operates over coupling graphs rather than gestural scores. In a coupling graph, coupling (temporal coordination) relations exist between gestures, but the specifics of gestures' relative timing and their respective activation durations are not yet set. At this level of representation, gestures are still temporally abstract. From the specifications present in the coupling graph, including gestures' parameters for intrinsic duration as well as intergestural coupling relations, the Coupled Oscillator Model (Nam \& Saltzman 2003; Nam 2007; Nam, Goldstein, \& Saltzman 2009) calculates gestural duration and timing and generates a gestural score.

Herein lies the complication when developing an analysis of extended gestural duration: the phonological component operates to produce a coupling graph, a level of representation at which gestural duration has not yet been determined. Therefore, durational information is not available to the phonological component and cannot be directly manipulated by it. Capturing extended gestural duration is not as simple 
as requiring a gesture to remain active for a longer-than-usual period of time, or for that gesture to overlap the gestures that follow it. This sort of direct manipulation of gestural duration is not possible in a coupling graph. Instead what is needed is some parameter of the extended gesture itself that is present underlyingly, and therefore within the coupling graph, that indicates that a gesture will extend in duration throughout a word once that duration is calculated by the Coupled Oscillator Model.

It is proposed that this crucial parameter within the coupling graph is the end phase specification of a gesture. A gesture's activation begins at the $0^{\circ}$ phase of an oscillator with which it is affiliated (its planning oscillator) and extends throughout the oscillatory cycle until it reaches a phase at which it automatically deactivates (roughly corresponding to the achievement of that gesture's target tract variable). This phase is specified for each automatically deactivating gesture; note that consonantal and vocalic gestures in the gestural score above are of different durations, indicating different end phase specifications. It is proposed here that some gestures are defective; that is, they lack this mechanism of automatic deactivation at a specified end phase and remain active beyond the phase at which typical gestures deactivate. In the case of nasal spreading, it is the gesture calling for opening of the velum that does not automatically deactivate. The result is extended duration of the velum opening gesture and the spread of nasality throughout a word.

The following figure contrasts the specifications of a typical gesture with those of a non-automatically deactivating gesture. A typical gesture has the ability to trigger itself at its own $0^{\circ}$ phase, indicated by the flag at its starting point, as well as the ability to deactivate itself at its specified end phase, indicated by the flag at its endpoint. A non-automatically deactivating gesture, on the other hand, has no specified end phase and therefore does not deactivate itself; this results in the gesture's extended duration.

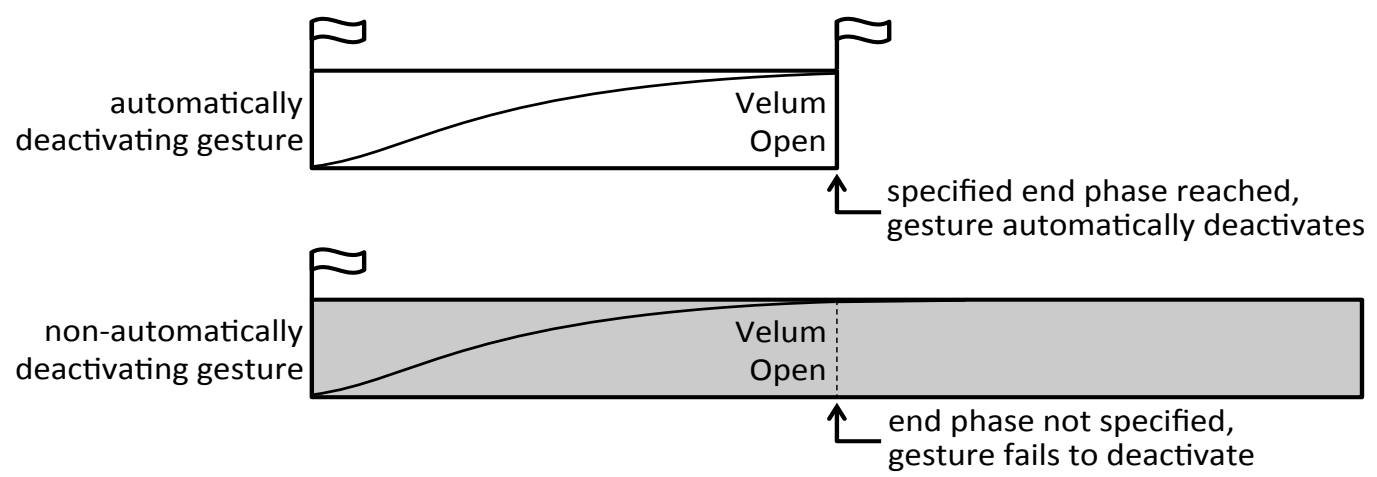

Figure (2): A typical gesture and non-automatically deactivating gesture. Flags indicate a gesture's ability to activate itself (assumed universal) and to deactivate itself at a specified phase.

This appeal to a gesture's ability or inability to deactivate itself allows for the representation of gestures as either having a typical or extended duration while still dealing only with information that is available within a coupling graph. When a coupling graph with a non-automatically deactivating velum opening gesture is input to the Coupled Oscillator Model, a gestural score in which that velum opening gesture does not deactivate and is thus active throughout an entire word will automatically result. Such a coupling graph, for the Warao form [mõjõ] 'cormorant,' is shown in the following figure. Lines between gestures indicate the presence of coupling relations; a solid line represents in-phase (synchronous) coupling, and a dashed arrow represents anti-phase (sequential) coupling (Browman \& Goldstein 2000). 


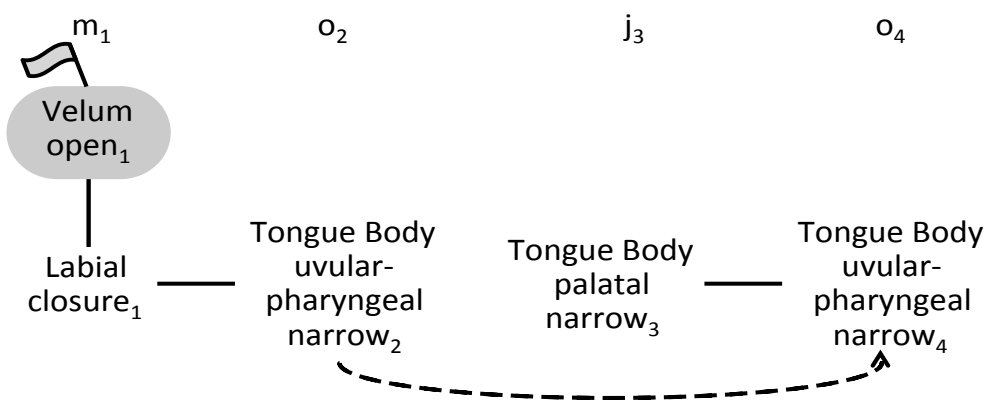

Figure (3): A coupling graph for the form [mõjõ ] 'cormorant.' The lack of a deactivation flag indicates a gesture's inability to automatically deactivate at a specified phase.

Note that the duration of the velum opening gesture for the $[\mathrm{m}]$, or any other gesture for that matter, is not specified in this coupling graph. However, the coupling graph will produce the gestural score for [mõjõ ] pictured in figure (1), with the activation of the velum opening gesture spanning the entire word, by virtue of the velum opening gesture not automatically deactivating the way all of the other gestures in the word do.

This approach to spreading as arising from a property of a trigger provides an interesting contrast with previous analyses. In ruled-based autosegmental analyses, spreading is traditionally the result of an iterative rule requiring association of a feature with an adjacent segment. Optimality Theory (Prince \& Smolensky 1993/2004; henceforth OT) is replete with constraints designed to capture spreading phenomena, including featural alignment constraints (Kirchner 1993; Akinlabi 1996), SPREAD constraints (Padgett 1995; Walker 1998/2000), and AGREE constraints (Baković 2000). In all of these analyses, the imperative to spread comes directly from the grammar rather than from a property of the trigger itself. In contrast, the gestural analysis presented here relies on the idea that a gesture does not deactivate itself due to its lack of a specified deactivation phase in order to trigger nasal spreading. A grammar may shape a language's inventory such that it contains a non-automatically deactivating velum opening gesture, but it does not directly manipulate gestural duration in order to generate patterns of nasal spreading.

\section{Transparency}

3.1 Transparency as Gestural Coactivation It is not uncommon for languages with nasal spreading to display transparency to that spreading. When this is the case, nasality spreads throughout a word, but a consonant within the span of nasality remains oral. Recall that in Tuyuca (Tucanoan; Colombia and Brazil) nasality may extend throughout an entire root morpheme, targeting vowels, glides, and liquids, but not voiceless obstruents (Barnes \& Takagi de Silzer 1976). Data in (2) is repeated in (3) for convenience.
a. [mĩpĩ] 'badger'
b. [w̃̃âi] 'demon'
c. [jũkã] 'yucca soup'
d. [jõsõ] 'bird'

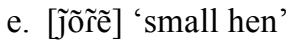

Piggott (1992) and (Walker 1998/2000) observe that obstruents are the only consonants that may surface as transparent in nasal spreading. Within featural theory, there is little consensus as to how this transparent behavior of obstruents should be analyzed, or even what the proper representation of transparency is. However, the adoption of gestural representations affords elegant resolutions of both of these issues. It is proposed that transparency to nasal spreading can be analyzed as the activation of an obstruent's velum closure gesture during the extended activation of a velum opening gesture, resulting in closure of the velopharyngeal port during the period of time in which the obstruent is being produced. In this way, transparent behavior in nasal spreading is limited to obstruents, matching crosslinguistic patterns. Additionally, obstruent orality within a nasal span can be captured without appealing to derivational opacity or to gapped representations in which a spreading [nasal] feature skips an obstruent segment. 
The incompatibility of obstruents with nasality is well established within the fields of phonetics and phonology. A stop is characterized by a buildup of air pressure during closure and an audible burst upon release. Fricatives depend on sufficient airflow through a narrow channel in order to produce the turbulence necessary for frication. Both of these acoustic events are dependent upon precise aerodynamic conditions of the vocal tract that are significantly disrupted by the opening of the velum. Therefore, many representations of obstruents in AP include both an oral constriction gesture and a velum closure gesture. This will ensure that there is a tight seal between the velum and the pharyngeal wall that will prevent the escape of air through the nasal cavity. While the neutral position of the velum (i.e., the position it assumes when not engaged by an active gesture) is assumed to be high enough to close the velopharyngeal port, the seal is not sufficiently tight for obstruents. Thus, tighter closure between the velum and pharyngeal wall must be achieved by a velum closure gesture that raises the velum relative to its neutral position. Articulatory data supports this theory. Numerous studies including Lubker (1968), Bell-Berti \& Hirose (1975), and BellBerti (1976) report raising of the velum during the production of oral stops relative to the height achieved during production of other oral segments.

In a Tuyuca word such as [mĩpĩ] 'badger,' the presence of nasality that extends throughout the word can be attributed to a non-automatically deactivating velum opening gesture that begins synchronously with the labial closure gesture of the $[\mathrm{m}]$. The $[\mathrm{p}]$ is represented as the combination of a labial closure gesture, a glottal opening gesture (to produce voicelessness), and a velum closure gesture. This is represented in the following coupling graph for [mĩpĩ].

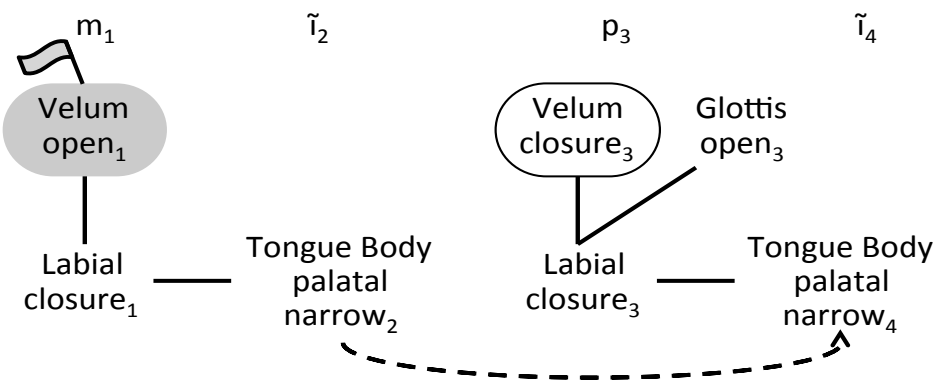

Figure (4): Coupling graph for [mĩpĩ] 'badger'

It is proposed that obstruent transparency to nasal spreading will obtain automatically from this coupling graph. No additional mechanism is necessary to ensure that the [p] of [mĩpĩ] is produced as oral rather than nasal, and no phonological relation exists between the transparent obstruent and the velum opening gesture that overlaps it. The coupling graph is specified such that when the gestural score and articulatory trajectories for this word are automatically generated, nasal spreading with a transparent obstruent will result. First, gestural durations and relative timing are set by the Coupled Oscillator Model, and the following gestural score is generated.

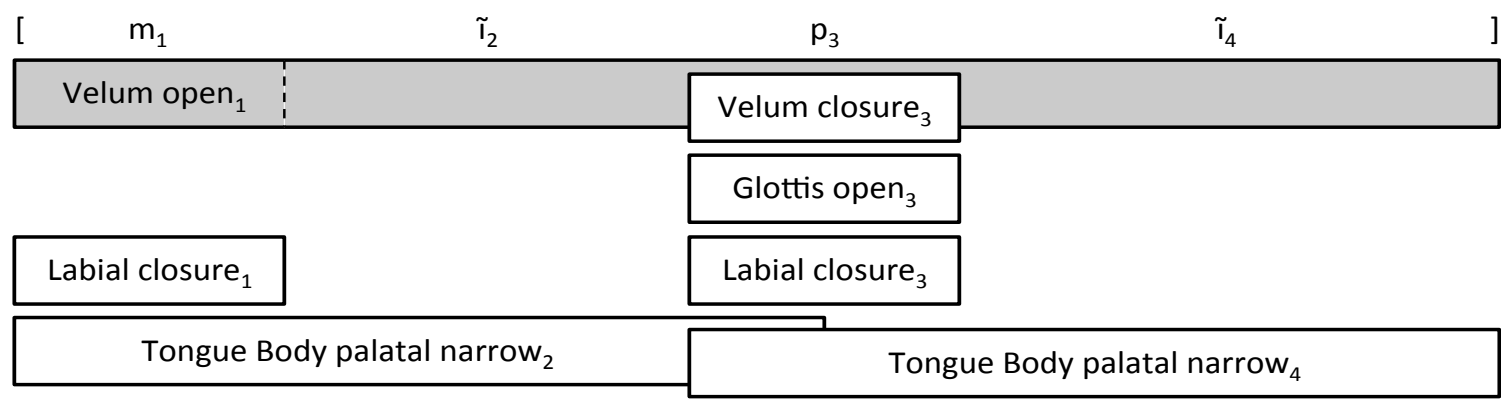

Figure (5): Gestural score for [mĩpĩ] 'badger'

Gestures are task-based units that, when active, enlist one or more articulators in order to achieve some articulatory goal. The velum opening gesture, due to its inability to deactivate itself, is active throughout the entire word, and thus exerts its influence over the velum during that time, requiring it to be open. 
However, gestures may also overlap one another to varying degrees, which has the potential to create conflict. Often, multiple simultaneously active gestures call for contradictory specifications of tract variables. Note that in the gestural score above the velum closure gesture of $[p]$ is active during a portion of the time that the velum opening gesture is also active. During this span of time, one gesture requires the velum to open, and the other requires it to close.

When simultaneously active gestures have competing goals for the same vocal tract variable (in this case, velum aperture), competition between gestures ensues and is resolved according to the workings of the Task Dynamic Model of speech production (Saltzman \& Munhall 1989). It is proposed that obstruent transparency in nasal spreading is the result of the resolution of this competition in favor of an obstruent's velum closure gesture. This is shown in the following figure, which shows the duration of the two competing gestures, the period of overlap during which they are coactive, and the resulting velum aperture that results from a competition for control of velum aperture in which the velum closure gesture emerges victorious.

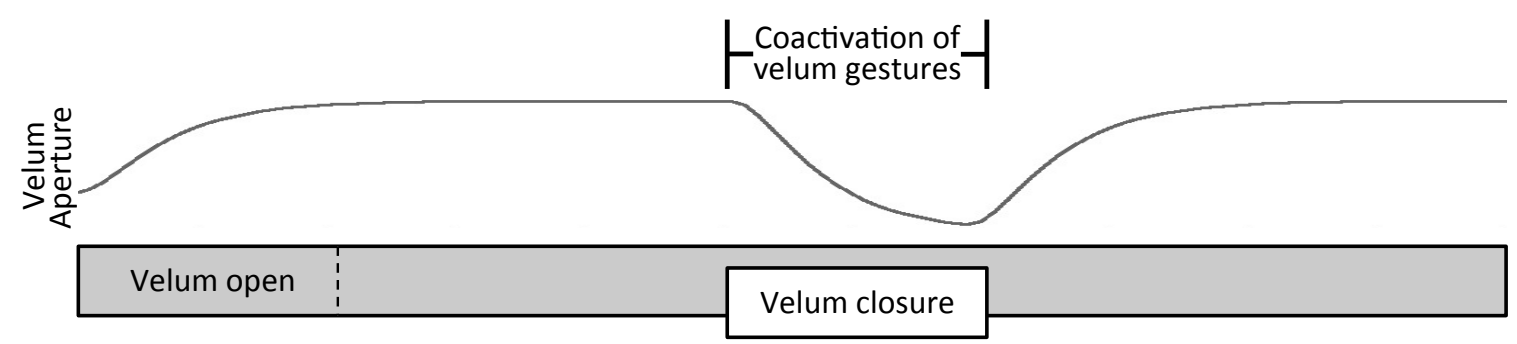

Figure (6): Two conflicting velum gestures and resulting velum aperture during their coactivation period

The activation of a velum opening gesture results in an increase in velum aperture, but later activation of a velum closure gesture causes the velum to close. Once the activation of the velum closure gesture has ended, the velum will open again according to the required specification of the still-active velum opening gesture. Thus nasality spreads throughout an entire word but appears to skip over obstruents that fall within the span of time that the velum opening gesture is active. This proposed coactivation transparency represents a novel way of thinking about what it means for a consonant to undergo nasal spreading versus behave transparently. In one sense, the transparent consonant $[\mathrm{p}]$ is an undergoer of nasal spreading, as the activations of its composite gestures are concurrent with the activation of a velum opening gesture. However, nasalization of this consonant has not occurred; the velum is closed and there is no nasal airflow due to the effect of the velum closure gesture.

Liquids, glides, and vowels are not represented in AP with an accompanying velum closure gesture because a loose seal of the velopharyngeal port is sufficient to achieve their less stringent oral airflow goals. The neutral position of the velum is sufficient to allow for the successful oral production of these sounds. Because these sounds do not include a velum closure gesture, they are not predicted by a gestural analysis to behave transparently in nasal spreading, unlike obstruents. When such non-obstruents are overlapped by the extended duration of a non-automatically deactivating velum opening gesture, they become nasalized. Obstruents, on the other hand, are assumed to include a velum closure gesture. This means not only that obstruents are the only consonants that may behave transparently to nasal spreading, but also that obstruents will always behave transparently whenever nasality is allowed to spread throughout an entire word. This is in line with crosslinguistic facts about nasal spreading, strengthening the appeal of the gestural analysis.

3.2 Alternative Analyses The analysis of obstruent transparency in nasal spreading as the result of the coactivation of two competing velum gestures is a radical departure from previous accounts of this phenomenon. Early analyses of spreading phenomena often rely on derivational opacity in order to capture instances of transparency. Piggott (1988), for instance, presents an analysis of nasal spreading in Guaraní in which the feature [+nasal] first spreads onto voiceless obstruents but is then denasalized by the rule [-voice] $\rightarrow$ [-nasal]. This sort of opacity, in addition to being difficult to implement within OT, represents a Duke of York derivation (Pullum 1976), with obstruent nasality following the path [-nasal] $\rightarrow[+$ nasal $] \rightarrow$ [-nasal]. The gestural account of obstruent transparency similarly represents obstruents as having 
undergone nasal spreading in the sense that they are overlapped by a non-automatically deactivating velum opening gesture. However, their surfacing as oral rather than nasal does not rely upon a subsequent step of repair as Piggott's featural account does; it falls out of the representations of velum gestures automatically.

Another proposed method of capturing transparency in nasal spreading involves allowing a [nasal] feature to spread beyond an obstruent without associating with it, resulting in a gapped configuration, as in Piggott (1992). However, later work including that by McCarthy (1994), Gafos (1996), and Ní Chiosáin \& Padgett (2001) claims that such configurations are universally ill-formed. According to this view, all spreading must respect strict segmental locality; skipping of segments in feature spreading is not permitted. The gestural coactivation account of transparency runs into no such problems with gapping and locality of spreading. Nasal spreading is the result of a single velum opening gesture extending in duration to overlap undergoing consonants and vowels. Even obstruents that appear to be skipped over in the spread of nasality are actually overlapped by this velum opening gesture.

Another key distinction between the gestural coactivation account of transparency and that of featural analyses is in the use of feature co-occurrence constraints restricting the spread of nasality onto obstruents. Phonetically grounded featural co-occurrence restrictions have long played a central role in analyses of transparency and blocking in spreading. Archangeli \& Pulleyblank (1994) account for segments that do not undergo spreading processes by proposing that the set of targets of a spreading rule may be limited to those that obey a feature co-occurrence restriction. Blocking arises when a rule that inserts an association line between a feature and a segment is prevented from applying to a restricted target. Transparency arises when a rule inserts an additional token of a spreading feature on the other side of the transparent segment, allowing that feature to avoid associating with a skipped-over target that would violate the rule's cooccurrence restriction.

Many OT analyses of transparency also rely on feature co-occurrence constraints. Notably among these are Optimal Domains Theory (Cole \& Kisseberth 1994, 1995) and Headed Span Theory (McCarthy 2004; O'Keefe 2005), in which segments are parsed into feature domains or spans. Blocking arises when a segment is prevented from being parsed into a domain/span due to the incompatibility of the domain's/span's spreading feature with one of the segment's other features. Transparency arises when a segment is parsed into a domain/span but does not bear its feature due to incompatibility. This incompatibility is captured by a feature co-occurrence constraint. Different rankings of the constraints calling for parsing into a domain/span, expression of the domain's/span's feature, and a relevant cooccurrence constraint will generate both transparency and blocking patterns.

A drawback of these accounts that utilize feature co-occurrence constraints to account for both transparency and blocking in spreading phenomena, whether rule- or constraint-based, is that they incorrectly predict that the sets of attested blocking and transparent segments are identical for a given spreading phenomenon. This is not the case for nasal spreading, in which all consonants are attested blockers but only obstruents are attested as transparent. Both Optimal Domains Theory and Span Theory can easily generate systems in which liquids or glides are transparent to nasal spreading despite such patterns being unattested. In contrast, the gestural account of nasal spreading makes no such prediction as transparency and blocking are treated as fundamentally different mechanisms. It is the underlying representation of obstruents as including a velum closure gesture that leads to their special status as the only attested transparent consonants in nasal spreading. Obstruent transparency in nasal spreading is the result of coactivation of velum opening and velum closure gestures, not an operation of the grammar. Cooccurrence restrictions crucially play no role in rendering obstruents transparent to nasal spreading.

\section{Blocking}

So far the gestural analysis of nasal spreading has focused on cases in which a velum opening gesture that does not automatically deactivate will remain active throughout the rest of a word. However, in many cases nasality does not spread fully but is instead checked by some class of oral consonants. Recall that in Warao nasal spreading proceeds through vowels, glides, and glottals but is blocked by liquids and obstruents (Osborn 1966). Data from (1) is repeated in (4) for convenience. 

a. [mõ̃̃õ] 'cormorant'
e. [hoñĩ̃ãau] 'turtle'
b. [inãw̃ããã] 'summer'
f. [ñãõte] 'he will come'
c. [mõãũ] 'give it to him'
g. [mõãũpu] 'give them to him!'
d. [mũũã̃ĩ] 'pink eye'
h. [ōõĩõro] 'kind of tree'

In languages with nasal spreading, it is posited that a velum opening gesture cannot deactivate itself. However, the velum opening gesture in each of the Warao forms in (4e-h), while still remaining active for a longer duration than a typical gesture would, does not remain active for the entirety of the word. Rather, the gesture remains active until it comes across a blocking consonant. Instead of automatically deactivating, the deactivation of a velum opening gesture is tied to the activation of the consonantal gesture that blocks it.

The set of blockers of nasal spreading in a language forms an implicational hierarchy roughly approximating the sonority hierarchy. This pattern was first reported by Schourup (1972), who noted that blocking of nasal spreading by glides implied blocking by liquids, which in turn implied blocking by obstruents. The incompatibility of nasalization with certain consonant types is also noted by Pulleyblank (1989), who utilizes phonetically based co-occurrence restrictions between the feature [nasal] and other consonantal features to capture blocking by liquids and obstruents. Walker (1998/2000) elaborates upon both of these points, claiming that the implicational hierarchy of consonantal blocking behavior is based on different consonants' incompatibility with nasalization, and proposing that this incompatibility be captured with a fixed ranking of co-occurrence constraints penalizing nasalized stops, fricatives, liquids, and glides. This is largely adopted in the gestural analysis of nasal spreading, though in this analysis the set of consonants that block nasal spreading will only be broken up into the classes of glides, liquids, and obstruents, without distinguishing between fricatives and stops.

The gestural account of nasal spreading also relies on co-occurrence restrictions to capture a nonautomatically deactivating velum opening gesture's inability to extend in duration beyond the gesture(s) of a following blocking consonant. It must be determined how best to define these gestural co-occurrence restrictions. Smith (forthcoming) makes use of gestural co-occurrence constraints that restrict incompatible gestures from being coupled to one another in a coupling graph. However, in the case of nasal spreading two gestures may not be anywhere near each other in a coupling graph, let alone coupled to one another, and yet may end up being active simultaneously in a gestural score due to one gesture's inability to automatically deactivate. Therefore, gestural co-occurrence constraints are of no use in capturing their incompatibility.

What is necessary in the case of spreading is some kind of restriction against the simultaneous activation of two incompatible gestures rather than coupling relations between them. However, gestural overlap is not represented in a coupling graph because gestural durations are not yet determined. Instead, a co-occurrence restriction between two gestures should take the form of a constraint that requires some specification to exist in the coupling graph that will result in no overlap between the gestures when the Coupled Oscillator Model generates its gestural score. A gestural analysis of blocking must account for the fact that in a form such as Warao [nãõte] 'he will come' the non-automatically deactivating velum opening gesture of the $[\mathrm{n}]$ may extend in duration to overlap the vowels [a] and [o], but not the obstruent [t] or anything that follows it. The generalization at the heart of this blocking behavior is that the activation of a blocking gesture causes or triggers the deactivation of the velum opening gesture.

It is proposed that this inhibitory relation between the two gestures is what must be represented in the coupling graph. Inhibition refers to a relation between two units in which the activation level of one unit detracts from the activation level of the other. In the case of blocking of nasal spreading, this inhibition relation is assumed to be unidirectional, with the activation of the blocking gesture inhibiting the activation of the velum opening gesture. Thus a velum opening gesture will deactivate when a consonant with which it is in an inhibitory relation is activated. In a coupling graph, an inhibitory relation between two gestures can be represented with the following notation:

Inhibited Gesture $\mathbf{x}$...... Inhibiting Gesture

Figure (7): An inhibitory relationship between gestures 
In the case of [nãõte], the velum opening gesture of [n] and the oral closure gesture of [t] must be in an inhibitory relation. This is represented in the following coupling graph:

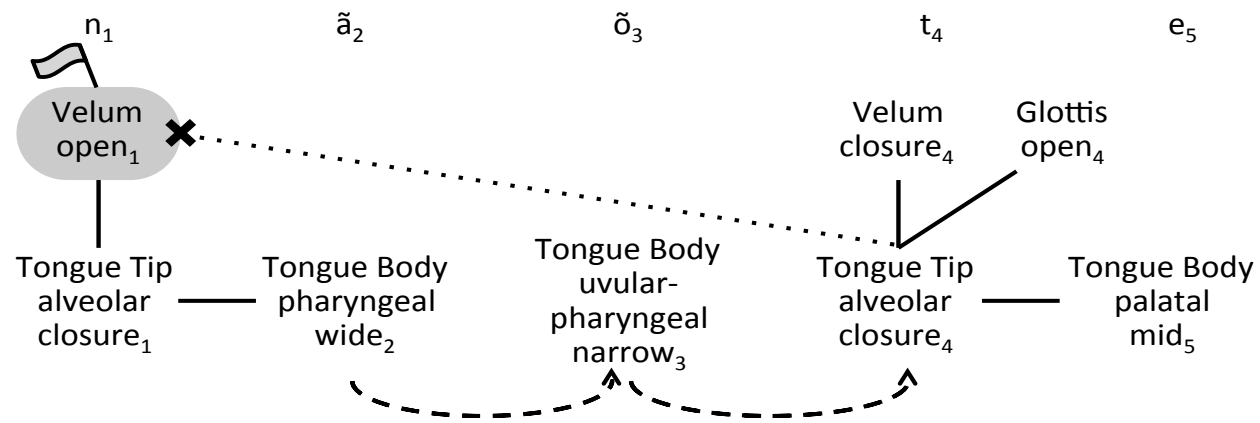

Figure (8): Coupling graph for [nãõte], with inhibitory gesture between the oral closure gesture of [t] and velum opening gesture of $[\mathrm{n}]$

The potential for extended duration of the velum opening gesture is represented by the gesture's inability to automatically deactivate. Its precise end time is not specified; instead it is specified that whenever the oral closure gesture of the [t] is activated, the velum opening gesture will deactivate. When this coupling graph, complete with inhibition relation between the velum opening gesture and its blocker, is input to the Coupled Oscillator Model, the result will be the following gestural score, in which the velum opening gesture extends only partially through the word such that the final syllable is not nasalized.

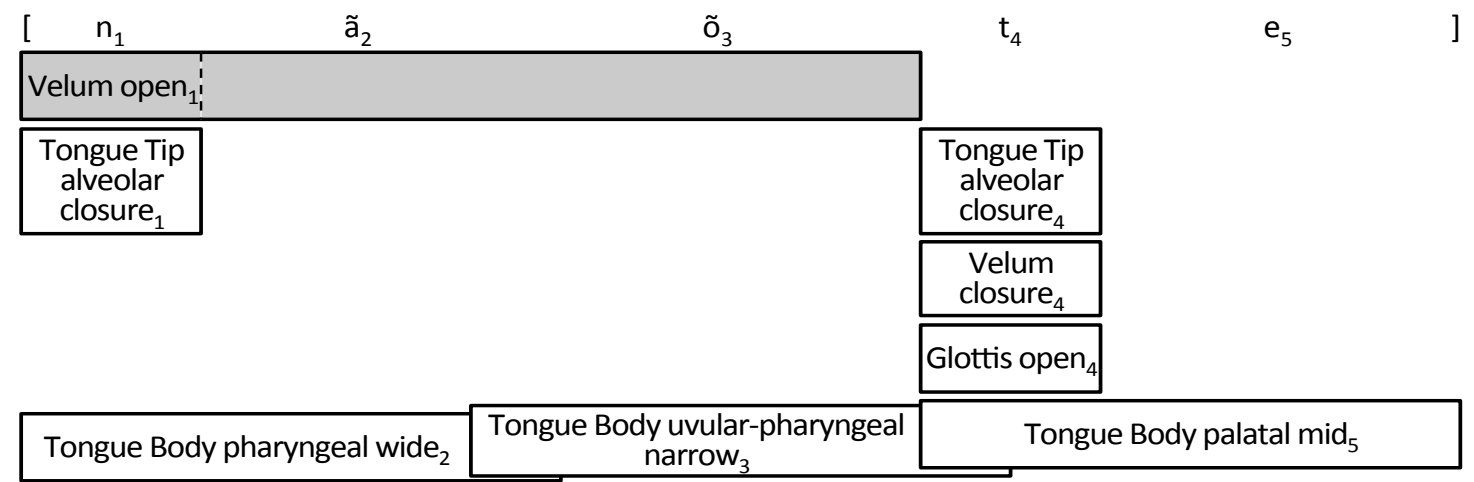

Figure (9): Gestural score for [nãõte] 'he will come,' with velum opening gesture extending through only part of the word

The implicational hierarchy of blockers can be accounted for by positing that a phonological grammar will require a non-automatically deactivating velum opening gesture to be in an inhibition relation with one of several possible gestural configurations. These are: (1) any oral constriction gesture, resulting in blocking by glides, liquids, and obstruents; (2) any oral gesture with a closed or critical constriction degree, resulting in blocking by liquids and obstruents; (3) any oral gesture accompanied by a velum closure gesture, resulting in blocking by obstruents. The second of these possibilities is exemplified in Warao. It is also possible for a grammar not to require a velum opening gesture to enter into an inhibition relation, as is the case in Tuyuca.

Now that the gestural analysis of blocking in nasal spreading has been laid out, it is clear what is meant by transparency and blocking being the results of fundamentally different mechanisms. Blocking is caused by a phonological relation, one of inhibition, that exists between a non-automatically deactivating velum opening gesture and a blocking gesture. This relation is taken into account when the Coupled Oscillator Model generates a gestural score. Transparency, on the other hand, relies on no such relation between gestures; it is the result of obstruents' inclusion of a velum closure gesture and the calculations of the Task Dynamic Model. This distinction is what allows the gestural analysis to more accurately reflect 
crosslinguistic patterns of nasal spreading, specifically that all consonants may act as blockers, but only obstruents may act transparently.

\section{Mixed Inventories of Nasals}

The gestural representation of nasal spreading is unique in that the imperative to spread nasality originates from a property of the phonological unit itself, and need not be rule- or constraint-driven (though the presence of the property of non-automatic deactivation may be constraint-driven). According to this view, the difference between a language that displays nasal spreading and one that does not is that the former has an inventory containing a non-automatically deactivating velum opening gesture while the latter has a typical automatically deactivating velum opening gesture. While the majority of languages can be analyzed as having either one or the other, it stands to reason that in other languages the two gestures should be able to turn up in the same phonological inventory and act as contrastive phonological units.

In fact, such languages do appear to exist. A number of languages spoken in and around Indonesia and Malaysia are reported to have two series of nasal consonants; one series triggers progressive nasal spreading, while the other does not. One such language is Acehnese (Malayo-Polynesian; Indonesia), in which some nasal consonants trigger nasal spreading among vowels, glides, and glottals, while others do not (data from Durie (1985) and Cowan (1981)).

\begin{tabular}{|c|c|}
\hline Non-Triggering Nasals & Triggering Nasals \\
\hline a. [miəb] 'suck' & $\overline{\text { d. [mãw̃ } \tilde{\Lambda}] \text { 'rose' }}$ \\
\hline b. [tinaj] 'to dwell' & 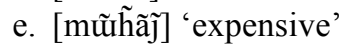 \\
\hline c. [mon] 'dew' & f. [nãw̃õy] 'soul' \\
\hline
\end{tabular}

Durie attributes this pattern to a distinction between plain nasals, which trigger the progressive nasal spreading that is common of Austronesian languages, and so-called 'funny' nasals, which do not trigger spreading. Similarly, McGinn (1982) and Coady \& McGinn (1982) propose a contrast in Rejang (MalayoPolynesian; Indonesia) between plain and 'barred' nasals, which contrast in their ability or inability to trigger nasal spreading.
Non-Triggering Nasals
Triggering Nasals
a. [dुamew] 'party'
d. [dुzamẽ w̃] 'guava fruit'
b. [tuyew] 'wait'
e. [tanẽn] 'hand'
c. [mon] 'cloud'
f. [mõnõã?] 'kill'

Patterns similar to those in Acehnese and Rejang, in which nasal spreading is triggered by one nasal consonant series and not another, have also been noted in Bukar-Sadong Land Dayak (Malayo-Polynesian; Indonesia, Malaysia) and Iban (or Sea Dayak, Malayo-Polynesian; Indonesia, Malaysia). The distinction between triggering and non-triggering nasals in these languages is easily captured by a gestural analysis. The only difference between these two nasal series lies in which velum opening gesture (automatically or non-automatically deactivating) accompanies a consonantal oral gesture. Plain nasals can be represented as an oral closure gesture accompanied by a non-automatically deactivating velum opening gesture. Words that include these gestures will exhibit nasal spreading. In contrast, the funny or barred nasals that do not trigger nasal spreading can be represented by an oral closure gesture accompanied by a typical automatically deactivating velum opening gesture.

The existence of these mixed nasal inventories are predicted by the gestural analysis of nasal spreading. When the imperative to spread nasality throughout a word comes from the phonological unit that is spreading, the analysis is quite straightforward. Nasality spreads when a form includes a nonautomatically deactivating velum opening gesture, and does not spread when a form includes an automatically deactivating velum opening gesture. In some cases, these gestures can exist within the same language. In order to capture such a pattern with an analysis in which a rule or constraint motivates spreading, however, one must rely heavily on indexation of forms. Because features, unlike gestures, are not spatiotemporal units, there is no way to specify a nasal feature as being of a spreading versus non- 
spreading type. Thus the adoption of a gestural representation of nasal spreading finds typological support in the mixed nasal inventories of the Malayo-Polynesian languages of Indonesia and Malaysia.

\section{Conclusion}

This paper has introduced a gestural analysis of nasal spreading as the result of non-automatic deactivation of a velum opening gesture and outlined some of the benefits of such an analysis. Specifically, adopting AP-based gestural representations affords a simple account of obstruent transparency in nasal spreading as the coactivation of a velum closure gesture during an interval in which a velum opening gesture of extended duration is active. In contrast, blocking is achieved via an inhibition relation between a blocking gesture and a non-automatically deactivating gesture. This separation of non-undergoing behavior into two fundamentally different mechanisms is what allows the gestural analysis of nasal spreading to correctly predict that while all consonants are attested blockers of nasal spreading, only obstruents are attested to act transparently. In addition, the gestural account of nasal spreading correctly predicts the existence of languages in which some nasals trigger spreading while others do not, exemplified by several Indonesian languages. The accurate predictions of the gestural analysis of nasal spreading are the major source of its strength over a featural account.

This paper has only addressed progressive (rightward) nasal spreading as extended gestural activation duration. Analyzing regressive (leftward) spreading of nasality as the result of gestural reordering and recoupling is the subject of ongoing work. Additional work also aims to extend the non-automatic gestural deactivation account to additional spreading phenomena, including those involving the spread of rounding, tongue root advancement and retraction, and pharyngealization.

\section{References}

Akinlabi, Akinbiyi. (1996). Featural Affixation. Journal of Linguistics, 32(2), 239-289.

Archangeli, Diana, \& Pulleyblank, Douglas. (1994). Grounded Phonology. MIT Press.

Baković, Eric. (2000). Harmony, Dominance and Control. Ph.D. Dissertation, Rutgers, The State University of New Jersey.

Barnes, Janet. (1996). Autosegments with Three-Way Lexical Contrasts in Tuyuca. International Journal of American Linguistics, 62(1), 31-58.

Barnes, Janet, \& Takagi de Silzer, Sheryl. (1976). Fonología del tuyuca. In Sistemas fonológicos de idiomas colombianos, Tomo III (pp. 123-137). Lomalinda: Editorial Townsend.

Bell-Berti, Fredericka. (1976). An Electromyographic Study of Velopharyngeal Function in Speech. Journal of Speech and Hearing Research, 19(2), 225-240.

Bell-Berti, Fredericka, \& Hirose, Hajime. (1975). Palatal activity in voicing distinctions: a simultaneous fiberoptic and electromyographic study. Journal of Phonetics, 3, 69-74.

Browman, Catherine P, \& Goldstein, Louis. (1986). Towards an Articulatory Phonology. Phonology Yearbook, 3, 219 252.

Browman, Catherine P, \& Goldstein, Louis. (1989). Articulatory gestures as phonological units. Phonology, 6(2), 201251.

Browman, Catherine P, \& Goldstein, Louis. (2000). Competing constraints on intergestural coordination and selforganization of phonological structures. Bulletin de la Communication Parlée, 5, 25-34.

Coady, James, \& McGinn, Richard. (1982). On the So-Called Implosive Nasals of Rejang. In R. Carle, M. Heinschke, P. W. Pink, C. Rost, \& K. Stadtlander (Eds.), Gava': Studies in Austronesian Languages and Cultures (pp. 437449). Berlin: Dietrich Reimer Verlag.

Cole, Jennifer, \& Kisseberth, Charles. (1994). An Optimal Domains Theory of Harmony. Studies in the Linguistic Sciences, 24(2), 101-114.

Cole, Jennifer, \& Kisseberth, Charles. (1995). Nasal Harmony in Optimal Domains Theory. In Proceedings of the Western Conference on Linguistics (pp. 44-58).

Cowan, H K J. (1981). An Outline of Achehnese Phonology and Morphology. Bulletin of the School of Oriental and African Studies, 44(3), 522-549.

Durie, Mark. (1985). A Grammar of Acehnese on the Basis of a Dialect of North Aceh. Dordrecht: Foris Publications.

Gafos, Adamantios Ioannis. (1996). The Articulatory Basis of Locality in Phonology. Ph.D. Dissertation, The Johns Hopkins University. 
Goldsmith, John A. (1976). Autosegmental Phonology. Ph.D. Dissertation, Massachusetts Institute of Technology. Published in 1979 by Garland Publishing.

Goldsmith, John A. (1990). Autosegmental and Metrical Phonology. Cambridge, MA: Blackwell Publishing.

Kirchner, Robert. (1993). Turkish Vowel Harmony and Disharmony: An Optimality Theoretic Account. In Rutgers Optimality Workshop I (ROW-I).

Lubker, James F. (1968). An Electromyographic-Cinefluorographic Investigation of Velar Function During Normal Speech Production. Cleft Palate Journal, 5, 1-18.

McCarthy, John. (1994). On Coronal "Transparency." Talk presented at the Annual Trilateral Phonology Weekend, University of California Santa Cruz.

McCarthy, John J. (2004). Headed spans and autosegmental spreading. unpublished manuscript, University of Massachusetts Amherst.

McCarthy, John J, \& Prince, Alan S. (1995). Faithfulness and Reduplicative Identity (No. 18) (pp. 249- 384).

McGinn, Richard. (1982). Outline of Rejang Syntax. Jakarta: Badan Penyelenggara Seri NUSA.

Nam, Hosung. (2007). Syllable-level intergestural timing model: Split-gesture dynamics focusing on positional asymmetry and moraic structure. In J. Cole \& J. I. Hualde (Eds.), Papers in Laboratory Phonology 9 (pp. 483506). Berlin: Walter de Gruyter.

Nam, Hosung, Goldstein, Louis, \& Saltzman, Elliot. (2009). Self-organization of syllable structure: a coupled oscillator model. In F. Pellegrino, E. Marisco, I. Chitoran, \& C. Coupé (Eds.), Approaches to Phonological Complexity (pp. 299 - 328). Berlin: Mouton de Gruyter.

Nam, Hosung, \& Saltzman, Elliot. (2003). A Competitive, Coupled Oscillator Model of Syllable Structure. In Proceedings of the 15th International Congress of Phonetic Sciences. Barcelona, Spain.

Ní Chiosáin, Máire, \& Padgett, Jaye. (2001). Markedness, Segment Realization, and Locality in Spreading. In L. Lombardi (Ed.), Segmental Phonology in Optimality Theory: Constraints and Representations (pp. 118-156). Cambridge University Press.

O'Keefe, Michael. (2005). Transparency in Span Theory. University of Massachusetts Occasional Papers in Linguistics 33: Papers in Optimality Theory 3. Amherst, MA.

Osborn, Henry A. (1966). Warao I: Phonology and Morphophonemics. International Journal of American Linguistics, $32(2), 108-123$.

Padgett, Jaye. (1995). Partial Class Behavior and Nasal Place Assimilation. In K. Suzuki \& D. Elzinga (Eds.), Proceedings of the Arizona Phonology Conference: Workshop on Features in Optimality Theory (pp. 145-183). Tucson: University of Arizona.

Pater, Joe. (2000). Non-uniformity in English secondary stress: the role of ranked and lexically specific constraints. Phonology, 17, 237-274.

Piggott, Glyne. (1988). A parametric approach to nasal harmony. In H. van der Hulst \& N. Smith (Eds.), Features, Segmental Structure and Harmony Processes (Part I) (pp. 131-167). Dordrecht: Foris Publications.

Piggott, G. L. (1992). Variability in Feature Dependency: The Case of Nasality. Natural Language and Linguistic Theory, 10, 33-77.

Prince, Alan, \& Smolensky, Paul. (1993). Optimality Theory: Constraint Interaction in Generative Grammar. Blackwell Publishing.

Pulleyblank, Douglas. (1989). Patterns of Feature Cooccurrence: The Case of Nasality. In S. L. Fulmer, M. Ishihara, \& W. Wiswall (Eds.), Proceedings of the Arizona Phonology Conference 2/Coyote Papers 9 (pp. 98-115).

Pullum, Geoffrey K. (1976). The Duke of York gambit. Journal of Linguistics, 12, 83-102.

Saltzman, Elliot, \& Munhall, Kevin G. (1989). A Dynamical Approach to Gestural Patterning in Speech Production. Ecological Psychology, 1(4), 333-382.

Schourup, Lawrence. (1972). Characteristics of Vowel Nasalization. Papers in Linguistics, 5, 530-548.

Smith, Caitlin. (2015). Morphological Consonant Mutation as Gestural Affixation. In Papers from the 50th Annual Regional Meeting of the Chicago Linguistic Society (CLS 50).

Walker, Rachel Leah. (1998). Nasalization, Neutral Segments, and Opacity Effects. Ph.D. Dissertation, University of California Santa Cruz. Publishing in 2000 by Garland Publishing. 